\title{
On higher twist chiral-odd pion generalized parton distributions
}

\section{B. Pire}

CPHT, École Polytechnique, CNRS, 91128 Palaiseau, France

\section{Szymanowski}

National Center for Nuclear Research (NCBJ), Warsaw, Poland

\section{S. Wallon*}

Laboratoire de Physique Théorique, Université Paris-Sud, CNRS, 91405 Orsay, France \& UPMC Univ. Paris 06, Faculté de Physique, 4 place Jussieu, 75252 Paris Cedex 05, France

Samuel.Walloneth.u-psud.fr

We define in the framework of light-cone collinear factorization method, the chiral-odd generalized parton distributions (GPDs) of a pseudoscalar hadron (such as the $\pi^{0}$ ) up to twist 6 . For that, we introduce the relevant matrix elements for 2-parton non-local operators, as well as matrix elements for 3-parton non-local correlators. Their detailed parametrization is fixed based on parity, charge conjugation and time reversal invariance. This leads to the introduction of 28 real GPDs, which are subject to constraints coming from the QCD equations of motion.

XXII International Workshop on Deep-Inelastic Scattering and Related Subject -DIS2014, April 28 - May 2, 2014, Warsaw, Poland

\footnotetext{
* Speaker.
} 

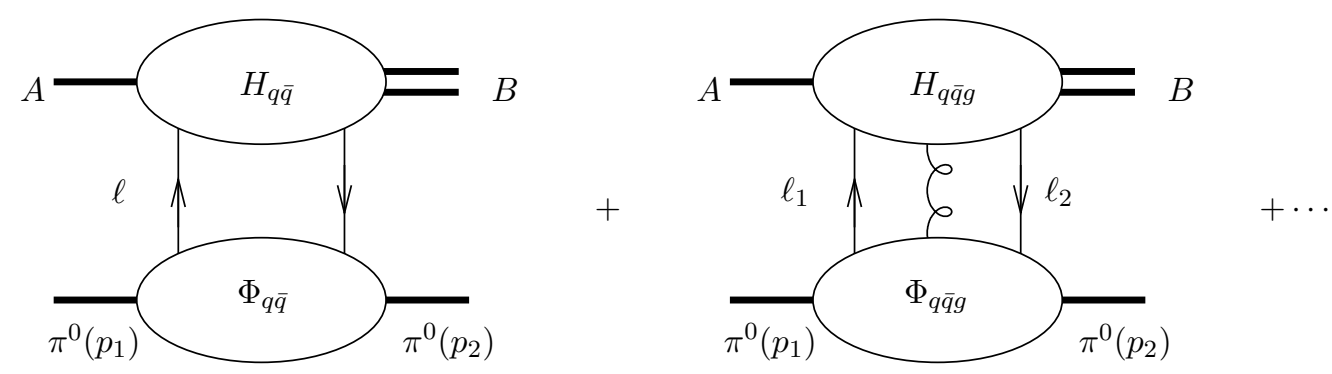

Figure 1: 2- and 3-parton correlators attached to a hard scattering amplitude in the process $A \pi^{0} \rightarrow B \pi^{0}$.

\section{Introduction}

The higher twist extension of the factorization properties of the leading twist amplitudes for exclusive exclusive reactions in the generalized Bjorken regime $[1,2,3]$ is a domain of intense recent research [4, 5, 6, 7, 8, 9, 10, 11]. We report here on an on-going study [12] of pion GPDs in the framework of the light-cone collinear factorization (LCCF), the generalization of the EllisFurmanski-Petronzio (EFP) method $[13,14,15]$ to the exclusive processes, which deals with the factorization in the momentum space around the dominant light-cone direction. Our first step is to provide a classification of chiral-odd $\pi^{0}$ GPDs. We restrict ourselves to 2-parton and 3parton correlators. We include the whole tower of twist contributions from 2 to 6 , but exclude any inclusion of pion mass effects, a question which has been successfully addressed recently $[16,17]$.

\section{Method}

Let us consider a hard exclusive process. For definiteness, we name as $Q$ the involved hard scale (e.g. the $\gamma^{*}$ 's virtuality in the case of deeply virtual Compton scattering (DVCS)). We here recall the basics of the LCCF in order to deal with amplitude of exclusive processes beyond the leading $Q$ power contribution. For definiteness, in view of the next sections, we illustrate the key concepts for the hard process $A \pi^{0} \rightarrow B \pi^{0}$ (where $A$ and $B$ denote generic initial and final states in kinematics where a hard scale allows for a partonic interpretation, for example $A=\gamma^{*}$ and $B$ a particle with a scalar or a tensor coupling), written in the momentum representation and in the $n \cdot A=0$ light-like gauge $\left(n^{2}=0\right)$, as

$$
\mathscr{A}=\int d^{4} \ell \operatorname{tr}[H(\ell) \Phi(\ell)]+\int d^{4} \ell_{1} d^{4} \ell_{2} \operatorname{tr}\left[H_{\mu}\left(\ell_{1}, \ell_{2}\right) \Phi^{\mu}\left(\ell_{1}, \ell_{2}\right)\right]+\ldots,
$$

where $H$ and $H_{\mu}$ are the coefficient functions with two parton legs and three parton legs, respectively, as illustrated in figure 1.

The major step out of the leading twist approximation is to expand the hard part $H(\ell)$ around the dominant $p$ direction:

$$
H(\ell)=H(y p)+\left.\frac{\partial H(\ell)}{\partial \ell_{\alpha}}\right|_{\ell=y p}(\ell-y p)_{\alpha}+\ldots,
$$

where $(\ell-y p)_{\alpha}=\ell_{\alpha}^{\perp}+(\ell \cdot p) n_{\alpha}$ allows one to extract higher twist contributions. One can see that this procedure introduces a $\ell^{\perp}$ and a $\ell \cdot p$ dependence inside the hard part which does not 
seem to fit with the standard collinear framework. To proceed toward a factorized amplitude, one performs an integration by parts to replace $\ell_{\alpha}^{\perp}$ by $\partial_{\alpha}^{\perp}$ and $\ell \cdot p$ by $\partial \cdot p$ acting on the soft correlator in coordinate space. This leads to new operators $\mathscr{O}^{\perp}$ and $\mathscr{O}^{-}$which contain transverse derivatives, such as $\bar{\psi} \partial^{\perp} \psi$ and longitudinal derivatives ${ }^{1}$ along $n$ denoted as $\partial_{n}^{\gamma} \equiv(\partial \cdot p) n^{\gamma}$ such as $\bar{\psi} \partial_{n}^{\gamma} \psi$ and thus to the necessity of considering additional non-perturbative correlators $\Phi^{\perp}$ and $\Phi^{-}$.

After these two steps, the amplitude takes the simple factorized form

$$
\begin{aligned}
\mathscr{A} & =\int_{-1}^{1} d y \operatorname{tr}\left[H_{q \bar{q}}(y) \Gamma\right] \Phi_{q \bar{q}}^{\Gamma}(y)+\int_{-1}^{1} d y \operatorname{tr}\left[H_{q \bar{q}}^{(\perp, p) \mu}(y) \Gamma\right] \Phi_{q \bar{q} \mu}^{(\perp,-) \Gamma}(y) \\
& +\int_{-1}^{1} d y_{1} d y_{2} \operatorname{tr}\left[H_{q \bar{q} g}^{(\perp, p) \mu}\left(y_{1}, y_{2}\right) \Gamma\right] \Phi_{q \bar{q} g \mu}^{(\perp,-) \Gamma}\left(y_{1}, y_{2}\right)+\cdots
\end{aligned}
$$

in which the two first terms in the r.h.s correspond to the 2-parton contribution and the last one to the 3-parton contribution. As usual the antiquark contribution is interpreted as the $[-1,0]$ part of this integral.

\section{Definitions}

Let us now construct the chiral-odd $\pi^{0}$ GPDs which parametrize the 2-parton and 3-parton correlators, taking into account constraints based on charge invariance, time invariance and parity invariance. The 2-partons correlators may be written as

$$
\left.\begin{array}{l}
\left\langle\pi^{0}\left(p_{2}\right)\left|\bar{\psi}(z)\left[\begin{array}{c}
\sigma^{\alpha \beta} \\
\mathbb{1} \\
i \gamma^{5}
\end{array}\right] \psi(-z)\right| \pi^{0}\left(p_{2}\right)\right\rangle=\int_{-1}^{1} d x e^{i(x-\xi) P \cdot z+i(x+\xi) P \cdot z} \times \\
{\left[-\frac{i}{m_{\pi}}\left(P^{\alpha} \Delta_{\perp}^{\beta}-P^{\beta} \Delta_{\perp}^{\alpha}\right) H_{T}+i m_{\pi}\left(P^{\alpha} n^{\beta}-P^{\beta} n^{\alpha}\right) H_{T 3}-i m_{\pi}\left(\Delta_{\perp}^{\alpha} n^{\beta}-\Delta_{\perp}^{\beta} n^{\alpha}\right) H_{T 4}\right.} \\
m_{\pi} H_{S} \\
0
\end{array}\right]
$$

twist $2 \& 4$

where each (real) GPD depends on the arguments $x, \xi, t$, and we underlined their twist content. We note that due to $\mathscr{P}$-parity invariance, there is no twist $3 \mathrm{GPD}$ associated with the $\gamma^{5}$ structure for the $\pi$ meson. This constraint will not survive in the nucleon GPD sector.

We now consider correlators involving the 3-parton and 2-parton (with transverse derivative).

\footnotetext{
${ }^{1}$ This completes the analysis performed in ref. [12].
} 
For the $\sigma^{\alpha \beta}$ structure, they read

$$
\begin{aligned}
& \left\langle\pi^{0}\left(p_{2}\right)\left|\bar{\psi}(z) \sigma^{\alpha \beta}\left\{\begin{array}{c}
i \overleftrightarrow{\partial_{\perp}^{\gamma}} \\
g A^{\gamma}(y)
\end{array}\right\} \psi(-z)\right| \pi^{0}\left(p_{1}\right)\right\rangle=\left\{\begin{array}{c}
\int \\
\int-1 \\
\int d^{3}\left[x_{1,2, g}\right] e^{i(x-\xi) P \cdot z\left(x_{1}+\xi\right)-i P \cdot y x_{g}+i P \cdot z\left(x_{2}-\xi\right)}
\end{array}\right\} \\
& \times\left[i m_{\pi}\left(P^{\alpha} g_{\perp}^{\beta \gamma}-P^{\beta} g_{\perp}^{\alpha \gamma}\right)\left\{\begin{array}{c}
T_{1}^{T} \\
T_{1}
\end{array}\right\}+\frac{i}{m_{\pi}}\left(P^{\alpha} \Delta_{\perp}^{\beta}-P^{\beta} \Delta_{\perp}^{\alpha}\right) \Delta_{\perp}^{\gamma}\left\{\begin{array}{c}
T_{2}^{T} \\
T_{2}
\end{array}\right\} \quad(\text { twist } 3 \& 5)\right. \\
& +i m_{\pi}\left(\Delta_{\perp}^{\alpha} g_{\perp}^{\beta \gamma}-\Delta_{\perp}^{\beta} g_{\perp}^{\alpha \gamma}\right)\left\{\begin{array}{c}
T_{3}^{T} \\
T_{3}
\end{array}\right\}+i m_{\pi}\left(P^{\alpha} n^{\beta}-P^{\beta} n^{\alpha}\right) \Delta_{\perp}^{\gamma}\left\{\begin{array}{c}
T_{4}^{T} \\
T_{4}
\end{array}\right\} \quad(\text { twist 4) } \\
& \left.+i m_{\pi}^{3}\left(n^{\alpha} g_{\perp}^{\beta \gamma}-n^{\beta} g_{\perp}^{\alpha \gamma}\right)\left\{\begin{array}{c}
T_{5}^{T} \\
T_{5}
\end{array}\right\}+i m_{\pi}\left(n^{\alpha} \Delta_{\perp}^{\beta}-n^{\beta} \Delta_{\perp}^{\alpha}\right) \Delta_{\perp}^{\gamma}\left\{\begin{array}{c}
T_{6}^{T} \\
T_{6}
\end{array}\right\}\right], \quad \text { (twist 5) }
\end{aligned}
$$

where

$$
\int d^{3}\left[x_{1,2, g}\right] \equiv \int_{-1+\xi}^{1+\xi} d x_{g} \int_{-1}^{1} d x_{1} \int_{-1}^{1} d x_{2} \delta\left(x_{g}-x_{2}+x_{1}\right)
$$

and $\overleftrightarrow{\partial_{\perp}^{\gamma}} \equiv \frac{1}{2}\left(\overrightarrow{\partial_{\perp}^{\gamma}}-\overleftarrow{\partial_{\perp}^{\gamma}}\right)$. The functions $T_{i}^{T}(i=1, \cdots 6)$ should be understood as $T_{i}^{T}(x, \xi, t)$, while $T_{i}(i=1, \cdots 6)$ denotes $T_{i}\left(x_{1}, x_{2}, \xi, t\right)$.

For the $\mathbb{1}$ structure, the correlators are defined as

$$
\begin{aligned}
\left\langle\pi^{0}\left(p_{2}\right)\left|\bar{\psi}(z) \mathbb{1}\left\{\begin{array}{c}
i \overleftrightarrow{\partial_{\perp}^{\gamma}} \\
g A^{\gamma}(y)
\end{array}\right\} \psi(-z)\right| \pi^{0}\left(p_{1}\right)\right\rangle & =\left\{\begin{array}{c}
1 \\
\int \\
-1 \\
\int d^{3}\left[x_{1,2, g}\right] e^{i(x-\xi) P \cdot z\left(x_{1}+\xi\right)-i P \cdot y x_{g}+i P \cdot z\left(x_{2}-\xi\right)}
\end{array}\right\} \\
& \times m_{\pi} \Delta_{\perp}^{\gamma}\left\{\begin{array}{c}
H_{S}^{T 4} \\
T_{S}
\end{array}\right\} . \quad \text { (twist 4) }
\end{aligned}
$$

For the $i \gamma^{5}$ structure, the correlators read

$$
\begin{aligned}
\left\langle\pi^{0}\left(p_{2}\right)\left|\bar{\psi}(z) i \gamma^{5}\left\{\begin{array}{c}
i \overleftrightarrow{\partial_{\perp}^{\gamma}} \\
g A^{\gamma}(y)
\end{array}\right\} \psi(-z)\right| \pi^{0}\left(p_{1}\right)\right\rangle & =\left\{\begin{array}{c}
\int d x e^{i(x-\xi) P \cdot z+i(x+\xi) P \cdot z} \\
-1 \\
\int d^{3}\left[x_{1,2, g}\right] e^{i P \cdot z\left(x_{1}+\xi\right)-i P \cdot y x_{g}+i P \cdot z\left(x_{2}-\xi\right)}
\end{array}\right\} \\
& \left.\times m_{\pi} \varepsilon^{\gamma n P \Delta_{\perp}}\left\{\begin{array}{c}
H_{P}^{T} \\
T_{P}
\end{array}\right\} . \quad \text { (twist } 4\right)
\end{aligned}
$$

We now consider correlators involving the 3-parton (with longitudinally polarized gluon) and 2-parton (with longitudinal derivative). We denote

$$
\partial_{n}^{\gamma} \equiv(\partial \cdot p) n^{\gamma} \quad \text { and } \quad A_{n}^{\gamma} \equiv(A \cdot p) n^{\gamma}
$$


For the $\sigma^{\alpha \beta}$ structure, they read

$$
\begin{aligned}
& \left\langle\pi^{0}\left(p_{2}\right)\left|\bar{\psi}(z) \sigma^{\alpha \beta}\left\{\begin{array}{c}
i \overleftrightarrow{\partial_{n}^{\gamma}} \\
g A_{n}^{\gamma}(y)
\end{array}\right\} \psi(-z)\right| \pi^{0}\left(p_{1}\right)\right\rangle=\left\{\begin{array}{l}
\int d x e^{i(x-\xi) P \cdot z+i(x+\xi) P \cdot z} \\
-1 \\
\int d^{3}\left[x_{1,2, g}\right] e^{i P \cdot z\left(x_{1}+\xi\right)-i P \cdot y x_{g}+i P \cdot z\left(x_{2}-\xi\right)}
\end{array}\right\} \\
& \times\left[\operatorname{im}_{\pi}\left(P^{\alpha} \Delta_{\perp}^{\beta}-P^{\beta} \Delta_{\perp}^{\alpha}\right) n^{\gamma}\left\{\begin{array}{c}
M_{1}^{-} \\
M_{1}
\end{array}\right\}\right. \text { (twist 4\&6) } \\
& \left.+i m_{\pi}^{3}\left(P^{\alpha} n^{\beta}-P^{\beta} n^{\alpha}\right) n^{\gamma}\left\{\begin{array}{c}
M_{2}^{-} \\
M_{2}
\end{array}\right\} \text { (twist 5) }+i m_{\pi}^{3}\left(n^{\alpha} \Delta_{\perp}^{\beta}-n^{\beta} \Delta_{\perp}^{\alpha}\right) n^{\gamma}\left\{\begin{array}{c}
M_{3}^{-} \\
M_{3}
\end{array}\right\} \text { (twist 6) }\right] \text {. }
\end{aligned}
$$

For the $\mathbb{1}$ structure, the correlators are defined as

$$
\begin{aligned}
\left\langle\pi^{0}\left(p_{2}\right)\left|\bar{\psi}(z) \mathbb{1}\left\{\begin{array}{c}
i \overleftrightarrow{\partial_{n}^{\gamma}} \\
g A_{n}^{\gamma}(y)
\end{array}\right\} \psi(-z)\right| \pi^{0}\left(p_{1}\right)\right\rangle & =\left\{\begin{array}{c}
1 \\
\int \\
-1 \\
\int d^{3}\left[x e^{i(x-\xi) P \cdot z+i(x+\xi) P \cdot z}\right.
\end{array}\right\} \\
& \times m_{\pi}^{3} n^{\gamma}\left\{\begin{array}{c}
H_{S}^{-} \\
M_{S}
\end{array}\right\} \quad \text { (twist 5) }
\end{aligned}
$$

Altogether, the 2- and 3-parton correlators lead to the introduction of 28 different GPDs. They are not independent, and the reduction to an independent set is not a simple task. The two basic tools to implement this reduction is firstly the QCD equations of motion and secondly the constraints usually denoted as $n$-invariance [13].

\section{Constraints from QCD equations of motion}

We start with the Dirac equation for the quark field

$$
\begin{aligned}
0= & \left\langle\pi^{0}\left(p_{2}\right)\left|(i \operatorname{DD} \psi)_{\alpha}(-z) \bar{\psi}_{\beta}(z)\right| \pi^{0}\left(p_{1}\right)\right\rangle \\
= & \left\langle\pi^{0}\left(p_{2}\right)\right|\left[i(\partial \cdot n)(\not p \psi)_{\alpha}(-z)+i(\partial \cdot p)(\hbar \hbar)_{\alpha}(-z)+\left(i \partial_{\perp} \psi\right)_{\alpha}(-z)\right. \\
& \left.+g\left(A_{\perp} \psi\right)_{\alpha}(-z)+g(A \cdot p)(\hbar \psi)_{\alpha}(-z)\right] \bar{\psi}_{\beta}(z)\left|\pi^{0}\left(p_{1}\right)\right\rangle .
\end{aligned}
$$

After a tedious but straightforward calculation of these five terms and demanding the vanishing of the contributions multiplying the four independent structures $\mathbb{P}_{\alpha \beta}, \mathbb{\Delta}_{\perp \alpha \beta}, t_{\alpha \beta}$ and $i \varepsilon^{\Delta_{\perp} P n \mu}\left(\gamma^{5} \gamma_{\mu}\right)_{\alpha \beta}$, we obtain the following four equations

$$
\begin{aligned}
& (x+\xi)\left(m_{\pi} H_{T 3}+m_{\pi} H_{S}\right)+\frac{\Delta_{\perp}^{2}}{2 m_{\pi}} H_{T}+2 m_{\pi} T_{1}^{T}+\frac{\Delta_{\perp}^{2}}{m_{\pi}} T_{2}^{T} \\
& +\frac{1}{2} \int_{-1+\xi}^{1+\xi} d x_{g} \int_{-1}^{1} d y \delta\left(x_{g}-x+y\right)\left(2 m_{\pi} T_{1}(y, x)+\frac{\Delta_{\perp}^{2}}{m_{\pi}} T_{2}(y, x)\right)=0,
\end{aligned}
$$




$$
\begin{aligned}
& (x+\xi)\left(\frac{P^{2}}{m_{\pi}} H_{T}-m_{\pi} H_{T 4}\right)+m_{\pi}\left(-\frac{1}{2} H_{S}+T_{3}^{T}+H_{S}^{T 4}\right) \\
& +\frac{m_{\pi}}{2} \int_{-1+\xi}^{1+\xi} d x_{g} \int_{-1}^{1} d y \delta\left(x_{g}-x+y\right)\left(T_{3}(y, x)+T_{S}(y, x)\right) \\
& -\frac{m_{\pi}}{2} \int_{-1+\xi}^{1+\xi} d x_{g} \int_{-1}^{1} d y \delta\left(x_{g}-x+y\right) M_{1}(y, x)-\frac{\Delta \cdot p}{2 m_{\pi}} H_{T}(x)-m_{\pi} M_{1}^{-}(x)=0, \\
& (x+\xi) m_{\pi} P^{2} H_{T 3}(x)+\frac{m_{\pi} \Delta_{\perp}^{2}}{2} H_{T 4}(x)-2 m_{\pi}^{3} T_{5}^{T}(x)-m_{\pi} \Delta_{\perp}^{2} T_{6}^{T}(x) \\
& -\frac{1}{2} \int_{-1+\xi}^{1+\xi} d x_{g} \int_{-1}^{1} d y \delta\left(x_{g}+y-x\right)\left(2 m_{\pi}^{3} T_{5}(y, x)+m_{\pi} \Delta_{\perp}^{2} T_{6}(y, x)\right) \\
& -\frac{m_{\pi}^{3}}{2} \int_{-1+\xi}^{1+\xi} d x_{g} \int_{-1}^{1} d y \delta\left(x_{g}-x+y\right) M_{S}(y, x)+\frac{m_{\pi}^{3}}{2} \int_{-1+\xi}^{1+\xi} d x_{g} \int_{-1}^{1} d y \delta\left(x_{g}-x+y\right) M_{2}(y, x) \\
& -\frac{\Delta \cdot p}{2} m_{\pi} H_{T 3}(x)+\frac{\Delta \cdot p}{2} m_{\pi} H_{S}(x)+m_{\pi}^{3} M_{2}^{-}(x)-m_{\pi}^{3} H_{S}^{-}(x)=0,
\end{aligned}
$$

and

$$
\begin{aligned}
& (x+\xi) H_{T 4}(x)-\frac{1}{2} H_{T 3}(x)+T_{4}^{T}(x)+H_{P}^{T}(x) \\
& +\frac{1}{2} \int_{-1+\xi}^{1+\xi} d x_{g} \int_{-1}^{1} d y \delta\left(x_{g}+y-x\right)\left(T_{4}(y, x)+T_{P}(y, x)\right) \\
& -\frac{1}{2} \int_{-1+\xi}^{1+\xi} d x_{g} \int_{-1}^{1} d y \delta\left(x_{g}+y-x\right) M_{1}(y, x)-\frac{\Delta \cdot p}{2 m_{\pi}^{2}} H_{T}(x)-M_{1}^{-}(x)=0 .
\end{aligned}
$$

A second set of four equations is obtained in an analogous way by considering the various correlators involved in the following equation coming from the Dirac equation on the antiquark field

$$
0=\left\langle\pi^{0}\left(p_{2}\right)\left|\psi_{\alpha}(-z)(i \mathrm{D} \bar{\psi})_{\beta}(z)\right| \pi^{0}\left(p_{1}\right)\right\rangle .
$$

This new set of equations is related by charge conjugation to the previous ones.

\section{Constraints from the $n$-invariance}

The physical amplitude for the process $A \pi^{0} \rightarrow B \pi^{0}$ should not depend on the arbitrary lightlike vector $n$, which is involved when defining the Fourier transform with respect to the light-cone momentum direction $p$ in order to define the various GPDs, as well as in the way one fixes the gauge. The requirement that $p \cdot n=1$ and $n^{2}=0$ effectively reduces to an invariance with respect to variations of $n_{\perp}$, i.e.

$$
\frac{d \mathscr{A}}{d n_{\perp}}=0
$$


As shown in detail in refs. [7, 8], for the case of the $\gamma^{*} \rightarrow \rho$ impact factor, such a requirement can be reduced, at twist-3 level, to a set of equations relating the various distribution amplitudes (DA) involved. Indeed, after proper use of Ward identities, one can effectively factorize out the hard Born contribution from eq. (5.1), in such a way that this equation leads to a set of constraints among the various non-perturbative correlators, i.e. in fine between the DAs themselves. Combining these equations with the one based on the QCD equations of motion, one can thus reduce the set of DAs to a minimal set, which are three independent DAs in the case of the twist 3 chiral-even $\rho$ meson.

A similar analysis can be performed for any physical amplitude involving the chiral-odd $\pi^{0}$ GPDs. The detailed study of the corresponding reduction to a minimal set of independent chiralodd GPDs for $\pi^{0}$ is under study.

\section{Acknowledgements}

This work is partly supported by the Polish Grant NCN No. DEC-2011/01/B/ST2/03915, the French-Polish collaboration agreement Polonium, the ANR "PARTONS", the PEPS-PTI "PHENODIFF", the Joint Research Activity Study of Strongly Interacting Matter (acronym HadronPhysics3, Grant Agreement n.283286) under the Seventh Framework Programme of the European Community and by the COPIN-IN2P3 Agreement.

\section{References}

[1] X.-D. Ji and J. Osborne, One-loop corrections and all order factorization in deeply virtual Compton scattering, Phys. Rev. D58 (1998) 094018, [hep-ph/9801260].

[2] J. C. Collins and A. Freund, Proof of factorization for deeply virtual Compton scattering in QCD, Phys. Rev. D59 (1999) 074009, [hep-ph/ 9801262$].$

[3] J. C. Collins, L. Frankfurt, and M. Strikman, Factorization for hard exclusive electroproduction of mesons in QCD, Phys. Rev. D56 (1997) 2982-3006, [hep-ph / 9611433 ].

[4] I. V. Anikin, B. Pire, and O. V. Teryaev, On the gauge invariance of the DVCS amplitude, Phys. Rev. D62 (2000) 071501, [hep-ph/ 0003203$].$

[5] I. V. Anikin and O. V. Teryaev, Wandzura-Wilczek approximation from generalized rotational invariance, Phys. Lett. B509 (2001) 95-105, [hep-ph/ 0102209 ].

[6] I. V. Anikin and O. V. Teryaev, Genuine twist 3 in exclusive electroproduction of transversely polarized vector mesons, Phys. Lett. $\mathbf{B 5 5 4}$ (2003) 51-63, [hep-ph/0211028].

[7] I. V. Anikin, D. Y. Ivanov, B. Pire, L. Szymanowski, and S. Wallon, On the description of exclusive processes beyond the leading twist approximation, Phys. Lett. B682 (2010) 413-418.

[8] I. V. Anikin, D. Y. Ivanov, B. Pire, L. Szymanowski, and S. Wallon, QCD factorization of exclusive processes beyond leading twist: $\gamma_{T}^{*} \rightarrow \rho_{T}$ impact factor with twist three accuracy, Nucl. Phys. $\mathbf{B 8 2 8}$ (2010) 1-68, [arXiv:0909.4090].

[9] I. V. Anikin, A. Besse, D. Y. Ivanov, B. Pire, L. Szymanowski, and S. Wallon, A phenomenological study of helicity amplitudes of high energy exclusive leptoproduction of the $\rho$ meson, Phys. Rev. D84 (2011) 054004, [arXiv:1105.1761].

[10] V. Braun and A. Manashov, Kinematic power corrections in off-forward hard reactions, Phys.Rev.Lett. 107 (2011) 202001, [arXiv: 1108 .2394]. 
[11] V. Braun and A. Manashov, Operator product expansion in QCD in off-forward kinematics: Separation of kinematic and dynamical contributions, JHEP 1201 (2012) 085.

[12] B. Pire, L. Szymanowski and S. Wallon, arXiv:1309.0083 [hep-ph].

[13] R. K. Ellis, W. Furmanski, and R. Petronzio, Unraveling Higher Twists, Nucl. Phys. B212 (1983) 29.

[14] A. V. Efremov and O. V. Teryaev, The transversal polarization in Quantum Chromodynamics, Sov. J. Nucl. Phys. 39 (1984) 962.

[15] O. V. Teryaev, Twist - three in proton nucleon single spin asymmetries, hep-ph/0102296.

[16] V. Braun, A. Manashov, and B. Pirnay, Finite-t and target mass corrections to DVCS on a scalar target, Phys.Rev. D86 (2012) 014003, [arXiv:1205.3332].

[17] V. Braun, A. Manashov, and B. Pirnay, Finite-t and target mass corrections to deeply virtual Compton scattering, Phys.Rev.Lett. 109 (2012) 242001, [arXiv:1209.2559]. 